\title{
Erratum: Mass Unspecific Supervised Tagging (MUST) for boosted jets
}

\author{
J.A. Aguilar-Saavedra, ${ }^{a}$ F.R. Joaquim ${ }^{b}$ and J.F. Seabra ${ }^{b}$ \\ ${ }^{a}$ Departamento de Física Teórica y del Cosmos, Universidad de Granada, \\ E-18071 Granada, Spain \\ ${ }^{b}$ Departamento de Fúsica and CFTP, Instituto Superior Técnico, Universidade de Lisboa, \\ Av. Rovisco Pais 1, 1049-001 Lisboa, Portugal \\ E-mail: jaas@ugr.es, filipe.joaquim@tecnico.ulisboa.pt, \\ joao.f.seabra@tecnico.ulisboa.pt
}

ERRATUM TO: JHEP03(2021)012

KEYWORDS: Jets

ARXIV EPRINT: 2008.12792

Because of an error in the production stage, the same figure was repeated throughout the paper. The originally published wrong file has been replaced online.

Open Access. This article is distributed under the terms of the Creative Commons Attribution License (CC-BY 4.0), which permits any use, distribution and reproduction in any medium, provided the original author(s) and source are credited. 